\title{
Indicadores de eficiência do uso da água e de nutrientes de clones de palma forrageira em condições de sequeiro no Semiárido brasileiro
}

\author{
Thieres George Freire Silva $\left({ }^{1 *}\right)$; Jorge Torres Araújo Primo ( $\left.{ }^{1}\right)$; Sérvulo Mercier Siqueira e Silva $\left({ }^{2}\right)$; \\ Magna Soelma Beserra de Moura ( $\left.{ }^{3}\right)$; Djalma Cordeiro dos Santos ( $\left.{ }^{2}\right)$; Maria da Conceição Silva $\left({ }^{2}\right)$; \\ José Eduardo Marques Araújo (')
}

(') Universidade Federal Rural de Pernambuco (UFRPE), Unidade Acadêmica de Serra Talhada (UAST), Caixa Postal, 63, 56900-000 Serra Talhada (PE), Brasil.

(2) Instituto Agronômico de Pernambuco (IPA), 56500-000 Arcoverde (PE), Brasil.

(3) Embrapa Semiárido, Caixa Postal, 23, 56302-970 Petrolina (PE), Brasil.

(*) Autor correspondente: thieres_freire@yahoo.com.br

Recebido: 6/ago./2013; Aceito: 3/mar./2014

\section{Resumo}

Objetivou-se avaliar a eficiência produtiva de três clones de palma forrageira resistentes à Cochonilha do Carmim, cultivados em condições de sequeiro no Semiárido brasileiro. Os clones avaliados foram IPA-Sertânia (IPA), Miúda (MIU) e Orelha de

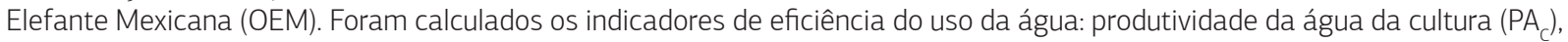
produtividade econômica da água (PEA) e eficiência do uso de nutrientes (EUN), todos com base na água precipitada (Prec.) e na evapotranspiração real da cultura (ETr). A ETr foi quantificada por meio do método do balanço de água no solo. Verificou-se que não houve diferença estatística entre a PA em base seca (MS) nos clones avaliados tanto em termos de Prec. como de ETr,

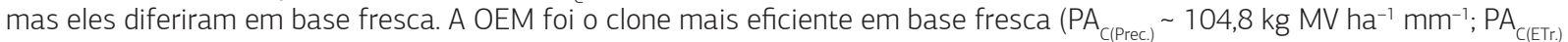
$\sim 112,1 \mathrm{~kg} \mathrm{MV} \mathrm{ha}^{-1} \mathrm{~mm}^{-1}$ ), seguido pelo clone IPA, que também se mostrou eficiente quanto a ETr $\left(\mathrm{PA}_{\mathrm{C(ETr} \text { ) }} \sim 101,1 \mathrm{~kg} \mathrm{MV} \mathrm{ha}^{-1}\right.$ $\mathrm{mm}^{-1}$ ). A MIU foi o clone menos eficiente para o Semiárido brasileiro. Não houve diferença da PEA (PEA (Prec.) $_{\text {(ETr.) }}$ 35,4 R\$ ha $\mathrm{hmm}^{-1} \mathrm{~mm}^{-1}$ $P_{E A} A_{(E T r)} \sim 39,5 R \$ h^{-1} \mathrm{~mm}^{-1}$ ) e da EUN, com exceção da eficiência do uso do magnésio com base na ETr, que foi maior para OEM (EUN $\left.{ }_{(\mathrm{Mg})} \sim 111,4 \mathrm{~g} \mathrm{ha}^{-1} \mathrm{~mm}^{-1}\right)$ e IPA $\left(\operatorname{EUN}_{(\mathrm{Mg})} \sim 77,4 \mathrm{~g} \mathrm{ha}^{-1} \mathrm{~mm}^{-1}\right)$, e do sódio com base na precipitação, que foi superior para os clones OEM (EUN ${ }_{(\mathrm{Mg})} \sim 4854,3 \mathrm{mg} \mathrm{ha}^{-1} \mathrm{~mm}^{-1}$ ) e MIU $\left(\mathrm{EUN}_{(\mathrm{Mg})} \sim 3383,6 \mathrm{mg} \mathrm{ha}^{-1} \mathrm{~mm}^{-1}\right)$.

Palavras-chave: evapotranspiração, Nopalea sp., Opuntia sp., produtividade econômica da água, rendimento.

\section{Water and nutrient use efficiency indicators of cactus pear clones in rainfed conditions in the Brazilian Semi-arid region}

\begin{abstract}
The objective was to evaluate the productive efficiency of three cactus pear clones, resistant to Cochineal Carmine, grown in rainfed conditions in the Brazilian semi-arid region. The evaluated clones were IPA-Sertânia (IPA), Miúda (MIU) and Orelha de Elefante Mexicana (OEM). The indicators of water use efficiency were calculated: crop water productivity (CWP), economic water productivity (EWP) and nutrient use efficiency (NUE), all based on rain (Prec.) and the real crop evapotranspiration (ETr). The ETr was quantified by the method of the soil water balance. It was found that the CWP on a dry basis (DB) did not differ

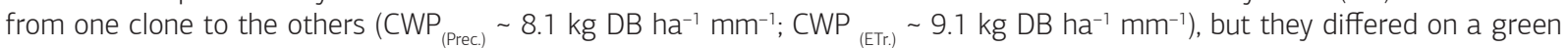

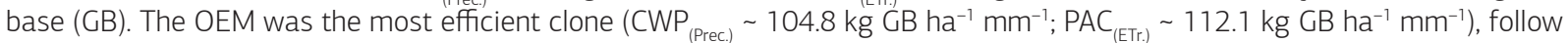
by the IPA concerning the $\operatorname{ETr}\left(C W P\left(\mathrm{ETT}_{\mathrm{r} .} \sim 101.1 \mathrm{~kg} \mathrm{~GB} \mathrm{ha-1}^{-1} \mathrm{~mm}^{-1}\right)\right.$. The MIU was least efficient clone for the Brazilian semi-arid region. There was no difference of PEA (PEA $A_{(\text {Prec.) }} \sim R \$ 35.4 \mathrm{ha}^{-1} \mathrm{~mm}^{-1} ; \mathrm{PEA}_{(\mathrm{ETr}) \text {. }} \sim \mathrm{R} \$ 39.5 \mathrm{ha}^{-1} \mathrm{~mm}^{-1}$ ) and EUN, with exception of magnesium use efficiency based on the ETr, which was higher for the OEM (EUN $\left.{ }_{(\mathrm{Mg})} \sim 111.4 \mathrm{~g} \mathrm{ha}^{-1} \mathrm{~mm}^{-1}\right)$ and IPA (EUN ${ }_{(\mathrm{M}}$ $\sim 77.4 \mathrm{~g} \mathrm{ha}^{-1} \mathrm{~mm}^{-1}$ ), and of the sodium based on the rain, which was superior for the OEM (EUN (Mg) $4854.3 \mathrm{mg} \mathrm{ha}^{-1} \mathrm{~mm}^{-1}$ ) and MIU (EUN (Mg) $3383.6 \mathrm{mg} \mathrm{ha}^{-1} \mathrm{~mm}^{-1}$ ) clones.
\end{abstract}

Key words: evapotranspiration, Nopalea sp., Opuntia sp., economic water productivity, yield. 


\section{INTRODUÇÃO}

No Semiárido brasileiro, devido a sua rusticidade e potencial de produção em condiçōes de pouca disponibilidade de água, a palma tornou-se um relevante recurso para a alimentação animal, com alta capacidade de oferta de forragem quando comparada à vegetação nativa da caatinga (Oliveira et al., 2010; Sales et al., 2009). Essa potencialidade está associada à ampla capacidade de adaptação dessa espécie, que tende a apresentar modificaçóes fisiológicas, anatômicas e estruturais em condições adversas (Nobel e Bobich, 2002).

Por causa da restrição hídrica nesse tipo de ambiente, o incremento da eficiência das culturas agrícolas pode ser feito por meio da adequação do uso de água no sistema de produção, seja alterando ou mesmo induzindo o aumento da produtividade das plantas (Di Paolo e Rinaldi, 2008; Jalota et al., 2006). Para isso é necessário conhecer a evapotranspiração da cultura, que varia em função da disponibilidade de água no solo; ambos estritamente relacionados com o rendimento das plantas, de modo que as relaçôes entre elas resultam num indicador denominado eficiência do uso da água (EUA) (Igbadun et al., 2006; Karam et al., 2007).

A EUA tem sido bastante utilizada na seleção de variedades mais tolerantes ao estresse hídrico (Igbadun et al., 2006; Ko e Piccinni, 2009). Por outro lado, o seu conhecimento também é de fundamental importância para o manejo da frequência e duração dos intervalos de reposição de água no solo, bem como para análise da viabilidade econômica da utilização de tecnologias de irrigação e de seus efeitos sobre o rendimento das culturas (Ali et al., 2007; Karam et al., 2007).

Existem vários indicadores de eficiência do uso da água (Pereira et al., 2012). Dentre eles podem-se citar a produtividade da água da cultura $\left(\mathrm{PA}_{\mathrm{C}}\right)$, a produtividade econômica da água (PEA) e a eficiência do uso de nutrientes (EUN). A PA $\mathrm{C}_{\mathrm{C}}$ considera a capacidade conversão da água em biomassa da cultura (Di Paolo e Rinaldi, 2008). Outro indicador de eficiência é a produtividade econômica da água, sendo de grande importância, uma vez que permite mensurar o incremento produtivo e o retorno econômico, gerados em função do uso da água no sistema de produção (Araya et al., 2011; Pereira et al., 2012). Finalmente, temos a eficiência do uso de nutrientes (EUN), que está sendo modificada no presente estudo em relação ao observado na literatura (Amaral et al., 2011), a fim de se analisar a capacidade de a cultura absorver nutrientes da soluçáo do solo com base na água consumida por ela ou recebida pelo sistema de produção, via irrigação ou precipitação.

$\mathrm{Na}$ literatura, informaçôes sobre a evapotranspiração e indicadores de eficiência da palma forrageira são raras (Consoli et al., 2013; Han e Felker, 1997), especialmente nas condições do semiárido brasileiro, o que fortalece a necessidade de suas determinaçôes visando melhorar a compreensão das respostas produtivas dessa espécie no clima e solo locais. Por sua vez, essas informaçôes podem variar em função de fatores como espécie vegetal, variedade, fases fenológicas, clima, solo, técnicas de manejo, sistemas de produção e época do ano (Azevedo et al., 2007; LópezUrrea et al., 2009; Suyker e Verma, 2009).

Assim, objetivou-se avaliar o desempenho de clones de palma forrageira resistentes à Cochonilha do Carmim cultivados em condiçôes de sequeiro no semiárido brasileiro a partir da obtençáo dos indicadores de eficiência do uso da água, como produtividade da água da cultura, produtividade econômica da água e de eficiência do uso de nutrientes, todos com base na precipitação pluviométrica e na evapotranspiraçáo real da cultura.

\section{MATERIAL E MÉTODOS}

Este estudo foi realizado em uma área cultivada com três clones de palma forrageira pertencente à Estação Experimental de Serra Talhada, do Instituto Agronômico de Pernambuco, localizada em Serra Talhada, Semiárido de Pernambuco $\left(7^{\circ} 59^{\prime} \mathrm{S}, 38^{\circ} 15^{\prime} \mathrm{O}\right.$ e $\left.431 \mathrm{~m}\right)$, que se caracteriza por um clima do tipo BSwh', de acordo com a classificação de Köppen, sendo os meses mais quentes aqueles com maiores níveis de chuva e os meses frios aqueles mais secos. O solo da área é classificado como do tipo Argissolo VermelhoAmarelo eutrófico franco arenoso, conforme classificação da Embrapa (1999). Os atributos químicos do solo antes do plantio podem ser observados na tabela 1.

Antes do plantio, em fevereiro de 2010, realizou-se aragem e gradagem do solo. Os clones foram cultivados em

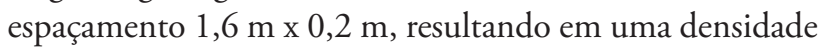
em torno de 31.250 plantas por hectare. A área foi dividida em nove parcelas experimentais, contendo quatro fileiras, cada uma com 20 plantas, ocupando uma área de $25,6 \mathrm{~m}^{2} \mathrm{e}$ área útil de $10,24 \mathrm{~m}^{2}$. Os tratamentos foram compostos por três clones de palma forrageira (IPA-200205/IPA-Sertânia do gênero Nopalea; IPA-100004/Miúda do gênero Nopalea; e IPA-200016/Orelha de Elefante Mexicana do gênero Opuntia). O ciclo produtivo foi conduzido em condiçóes de sequeiro, de modo que das nove parcelas experimentais utilizadas três foram compostas por cada clone, dispostas num delineamento em blocos ao acaso, com três repetiçôes.

Foram realizadas capinas manuais, aplicaçóes de herbicida e de inseticidas, quando necessário, para manutenção da área livre de plantas espontâneas e de pragas, respectivamente.

Tabela 1. Atributos químicos do solo da área experimental cultivado com clones palma forrageira em condiçôes de sequeiro no Semiárido pernambucano

\begin{tabular}{cccccc}
$\mathbf{p H}$ & $\mathbf{P}$ & $\mathbf{C a}$ & $\mathbf{A l}$ & $\mathbf{N a}$ & $\mathbf{K}$ \\
$\mathbf{H}_{2} \mathbf{O}$ & $\mathbf{m g ~ d m}^{-3}$ & & \multicolumn{2}{c}{$\mathbf{c m o l c ~}_{\mathbf{~ d m}}^{-3}$} & \\
6,7 & $>40,3$ & 3,25 & 0,0 & 1,52 & 0,45 \\
\hline
\end{tabular}


A adubação de $130 \mathrm{~kg}$ de $\mathrm{N} \mathrm{ha}^{-1}$ foi dividida em três vezes ao longo do ciclo produtivo. O herbicida aplicado foi o Diuron a $0,5 \%$ e o inseticida folisuper a $0,2 \%$ para realizar o controle fitossanitário. O primeiro corte da cultura foi realizado com dois anos de ciclo, em março de 2012.

Entre 20 de outubro de 2010 e 3 de março de 2012 foi realizado o monitoramento do conteúdo de água no solo por meio de uma sonda capacitiva (Diviner 2000, Sentek Pty Ltda., Austrália). A sonda possui um sensor portátil constituído por um sistema de aquisição de dados, que uma vez inserido em um tubo de acesso, instalado no solo, fornece de maneira rápida o conteúdo de água a cada $0,1 \mathrm{~m}$. Os tubos foram enterrados em prumo até uma profundidade de 0,9 m e, em seguida, foram vedados nas extremidades, para impedir a entrada de água. Para o uso da sonda capacitiva fez-se necessária a sua calibração nas condiçōes edáficas do experimento, conforme recomendaçóes do fabricante (Sentek, 2000; 2001). Os tubos de acesso foram instalados nas nove parcelas entre as fileiras centrais dos três clones, a uma distância de $0,1 \mathrm{~m}$ das plantas.

As condiçóes meteorológicas reinantes ao longo do ciclo da palma forrageira foram monitoradas por meio de uma PCD (plataforma de coleta de dados automatizada), localizada a aproximadamente $700 \mathrm{~m}$ da área experimental, pertencente à Agência Pernambucana de Água e Clima - APAC, equipada com um sistema de aquisição que permite o armazenamento dos dados medidos por sensores de temperatura $\left(t,{ }^{\circ} \mathrm{C}\right) \mathrm{e}$ umidade relativa do ar (UR, \%), pluviômetros (P, mm), radiação solar $\left(\mathrm{Rg}, \mathrm{W} \mathrm{m} \mathrm{m}^{-2}\right)$, velocidade $\left(\mathrm{u}, \mathrm{m} \mathrm{s}^{-1}\right)$ e direção do vento (DV). Esses dados são disponibilizados para intervalos de três horas, no site do Centro de Previsão do Tempo e Estudos Climáticos - CPTEC/INPE. Os dados foram utilizados também para estimativa da evapotranspiração de referência (ETo), por meio do método de Penman-Monteith parametrizado conforme o boletim 56 da FAO (Allen et al., 1998). Para o cálculo da evapotranspiração de referência foi utilizado o software REF-ET.

A biomassa foi obtida por meio da amostragem de dois cladódios do terço médio da parte aérea de duas plantas por clone de palma forrageira, de modo que uma vez pesados (para obtenção da massa fresca) em balança de precisão, fragmentados e acondicionados em sacos de papel devidamente identificados, foram conduzidos a uma estufa de ventilação forçada a $65^{\circ} \mathrm{C}$, do Laboratório de Análises de Alimentos Vegetais e Animais da Unidade Acadêmica de Serra Talhada - UAST, Universidade Federal Rural de Pernambuco - UFRPE, até obter-se massa seca constante. Os valores de produtividade em matéria fresca e seca foram extrapolados assumindo-se o número médio de cladódios por planta e a densidade de plantas por hectare na ocasião da colheita.

A partir das amostras de biomassa seca foram determinadas a concentraçáo de macronutrientes e de micronutrientes na planta, conforme metodologia descrita em Silva e Queiroz
(2002), por meio do envio das amostras aos laboratórios de Solos e Nutrição de Plantas e de Nutrição Animal da Embrapa Semiárido, situada no município de Petrolina, PE.

A evapotranspiraçáo dos clones de palma forrageira (ETr) foi quantificada por meio do resíduo do balanço de água no solo (BAS), para intervalos de 14 dias (resultando 37 períodos nos 499 dias), usando os dados do conteúdo de água $(\theta)$ e as propriedades físico-hídricas do solo. No BAS foram quantificados os componentes: variação de armazenamento $(\triangle \mathrm{A})$, precipitação $(\mathrm{P})$, evapotranspiração (ET), escoamento superficial (R) e densidade de fluxo $(\mathrm{Q})$, em um intervalo de 14 dias, para uma camada de solo de 0,60 $\mathrm{m}$ de profundidade. Considerou-se que a área experimental possuía baixa declividade $(<2 \%)$, de modo que os ganhos e perdas de água por escoamento subsuperficial foram desprezíveis ou se anularam. A variação do armazenamento de água no solo $(\Delta \mathrm{A})$ foi obtida mediante a integração dos valores de lâmina de água a cada $0,10 \mathrm{~m}$ resultantes dos dados de $\theta$. A precipitação (P) foi monitorada utilizandose os dados da estação meteorológica. $\mathrm{O}$ escoamento superficial (R) foi estimado utilizando-se o método Número da Curva - CN, proposto pela SCS-USDA, adotando-se o valor de $\mathrm{CN}$ de 75 , que representa uma condição de solo com taxa de infiltraçấo moderada quando completamente úmido e plantio em fileiras com curvas de nível de boa condição hidrológica. Q foi determinado através da base limite inferior do perfil do solo (0,60 $\mathrm{m}$ de profundidade) utilizando-se dados do potencial total de água da camada logo acima $(0,50 \mathrm{~m})$ e abaixo $(0,70 \mathrm{~m})$ e a equação BuckinghamDarcy (Libardi, 2005). As propriedades físico-hídricas do solo foram obtidas para a área experimental ao longo do perfil do solo por meio da aplicação em campo do método do perfil instantâneo, conforme Libardi (2005), em que se determinou a curva de retenção de água, ajustando-se o modelo exponencial e a condutividade hidráulica do solo não saturado aos valores de $\theta$.

Como as medidas de umidade do solo $(\theta)$ foram iniciadas após oito meses do plantio (20 de outubro de 2010), para se obter a ETr acumulada durante o crescimento da cultura, a fim de se calcular os indicadores de eficiência, a ETr foi estimada para intervalos de 14 dias, anterior a 20 de outubro de 2010, utilizando-se os valores da razáo P/ ETr (sendo de $-0,91$ para a IPA, $-0,92$ para a MIU e de $-0,79$ para a OEM), determinados utilizando-se os dados dos 37 períodos de balanço de água no solo e os dados de precipitação obtidos desde 15 de fevereiro de 2010, quando se iniciou o ciclo, até 19 de outubro de 2010.

A eficiência do uso da água foi avaliada por meio da produtividade da água da cultura $\left(\mathrm{PA}_{\mathrm{C}}, \mathrm{kg} \mathrm{m}^{-3}\right)$, a produtividade econômica da água (PEA, $\mathrm{R} \$ \mathrm{~m}^{-3}$ ) e em termos de eficiência do uso de nutrientes (EUN(w), $\left.\mathrm{kg} \mathrm{m}^{-3}\right)$. Os cálculos foram realizados assumindo-se os dados de biomassa, concentração de nutrientes, precipitação e evapotranspiração 
acumulados para todo o ciclo produtivo em condiçôes de sequeiro.

$\mathrm{A} \mathrm{PA}_{\mathrm{C}}$, que se refere à capacidade de a água utilizada ou recebida pela cultura converter fotoassimilados em biomassa total acumulada, foi determinada para a palma forrageira em condiçóes de sequeiro. Para isso, foi considerada a relação entre o rendimento e a evapotranspiração real acumulada ou a precipitação recebida pela cultura ao longo do tempo (Geerts e Raes, 2009):

$$
\mathrm{PA}_{\mathrm{C}}=\frac{\mathrm{Y}}{\sum \mathrm{ETr} \text { ou } \sum(\mathrm{P})}
$$

em que $\mathrm{PA}_{\mathrm{C}}$ é expressa em $\mathrm{kg} \mathrm{ha}^{-1} \mathrm{~mm}^{-1}$; Y é o rendimento da cultura em base fresca e base seca $\left(\mathrm{kg} \mathrm{ha}^{-1}\right)$; ETr é a evapotranspiraçáo real de um clone $(\mathrm{mm})$; e $\mathrm{P}$ é a precipitaçáo pluviométrica $(\mathrm{mm})$, todos dados acumulados ao longo do ciclo da cultura.

A produtividade econômica da água (PEA) foi calculada com base no ganho econômico (GE) por unidade de área, o que permite conhecer qual foi o retorno econômico de cada unidade de água utilizada pela cultura ou recebida pelo sistema de plantio. Assim, utilizou-se (Araya et al., 2011):

$$
\mathrm{PEA}=\frac{\mathrm{GE}}{\sum \mathrm{ETr} \text { ou } \sum(\mathrm{P})}
$$

em que GE é o ganho econômico por unidade de área, em $\mathrm{R} \$ \mathrm{ha}^{-1}$, que considerará o valor pago por unidade de cladódio de palma forrageira.

A eficiência do uso de nutriente (EUN(w)) foi adaptada da literatura, que assume a habilidade de a planta absorver os nutrientes da solução do solo (Amaral et al., 2011). No presente estudo, essa eficiência foi calculada com base na evapotranspiração real acumulada $\left(\sum \mathrm{ETr}, \mathrm{mm}\right)$ ou na precipitação $(\mathrm{P}, \mathrm{mm})$ recebida pela cultura. Esse indicador foi obtido para todos os macronutrientes e micronutrientes, considerando a sua concentração na planta $([\mathrm{Nu}])$ e a biomassa acumulada $(\mathrm{Y})$, de modo a se estabelecer a capacidade de absorção de um determinado nutriente da soluçấo do solo da planta em relação à quantidade de água utilizada pela cultura ou recebida pelo sistema de produção. Do mesmo modo que para os demais indicadores, o mesmo foi determinado para os três clones. Assim, tem-se que:

$$
\operatorname{EUN}(\mathrm{w})=\frac{\mathrm{Y} \cdot[\mathrm{Nu}]}{\sum \mathrm{ETr} \text { ou } \sum(\mathrm{P})}
$$

em que EUN(w) é expressa em $\mathrm{g} \mathrm{ha}^{-1} \mathrm{~mm}^{-1}$ ou $\mathrm{mg} \mathrm{ha}^{-1}$ $\mathrm{mm}^{-1}$; w se refere ao nutriente em análise $(\mathrm{N}, \mathrm{P}, \mathrm{K}, \mathrm{Mg}$ etc.); Y é o rendimento da cultura, em $\mathrm{kg} \mathrm{ha}^{-1}$; e $[\mathrm{Nu}]$ é a concentraçáo do nutriente na amostra analisada, dada em $\mathrm{g} \mathrm{kg}^{-1}$ ou $\mathrm{mg} \mathrm{kg}^{-1}$.

Os dados experimentais foram tratados por meio de estatística descritiva, na qual foram calculados os valores médios, desvios padrão e coeficientes de variação. A análise estatística experimental foi aplicada para realizar comparaçôes entre os três clones de palma forrageira. Nesse caso foi realizada análise de variância e, uma vez significativa pelo teste $\mathrm{F}$, assumindo-se o modelo que descreve o delineamento em blocos ao acaso, as médias foram submetidas ao teste de médias de Tukey, ao nível de 5\% de significância.

\section{RESULTADOS E DISCUSSÃO}

Na figura 1 é apresentada a comparação entre as variáveis meteorológicas e as normais climatológicas do município de Serra Talhada. Os anos 2010, 2011 e início de 2012, que compreenderam os 745 dias após o plantio, apresentaram condiçôes meteorológicas com valores atípicos em relação à normal climatológica. Percebe-se que a temperatura média do ar esteve ligeiramente acima da média da normal climatológica $\left(25,83^{\circ} \mathrm{C}\right.$ versus $\left.25,23^{\circ} \mathrm{C}\right)$ e que a umidade relativa do ar esteve abaixo $(60,21 \%$ versus $63,43 \%)$. Essas condiçôes promoveram uma alta demanda atmosférica durante o período ( $3777 \mathrm{~mm}$ versus $2844 \mathrm{~mm}$ ), gerando um alto déficit hídrico, embora a precipitação tenha sido

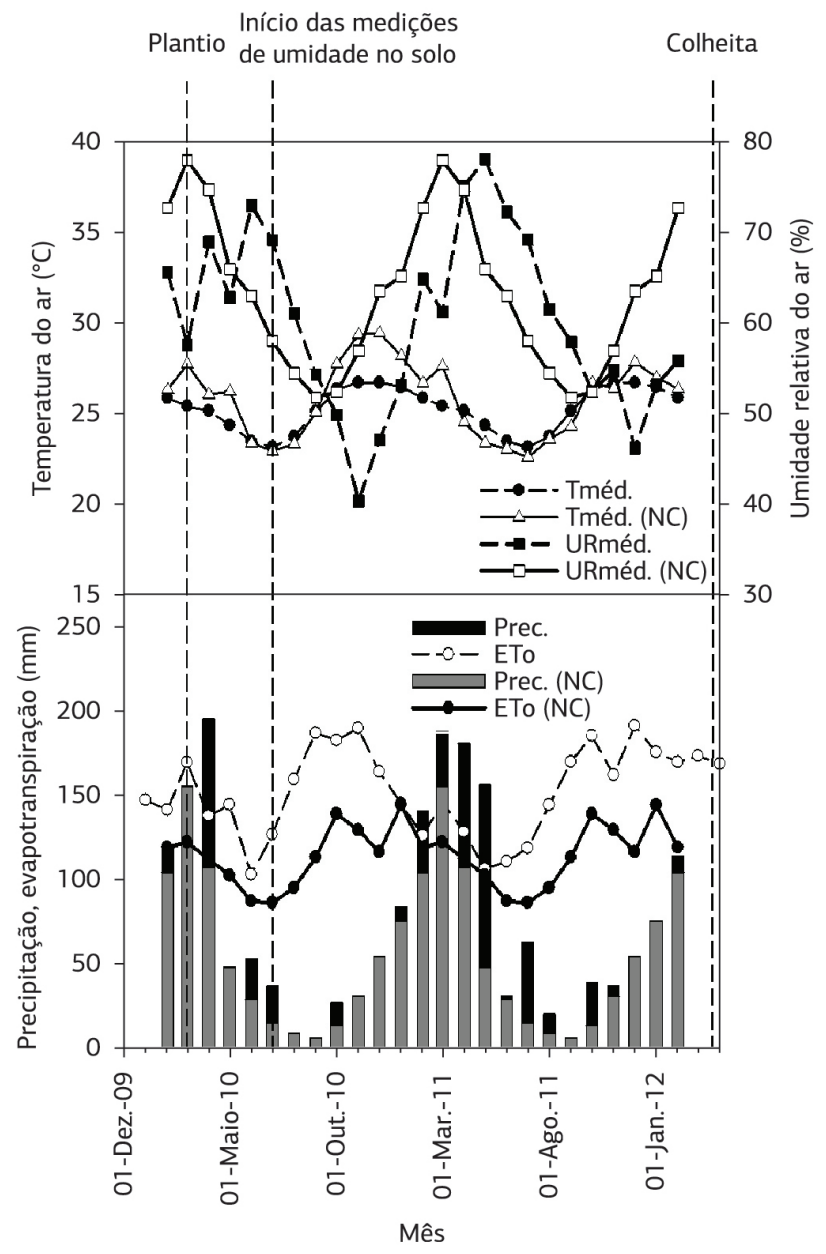

Figura 1. Condiçôes meteorológicas e normais climatológicas (NC) das variáveis meteorológicas e da demanda atmosférica ao longo do ciclo produtivo dos clones de palma forrageira em Serra Talhada, Semiárido brasileiro. 
acima da normal climatológica na maioria dos meses (1582 $\mathrm{mm}$ versus $1388 \mathrm{~mm}$ ).

Essas condiçôes conferiram à Orelha de Elefante Mexicana a maior produtividade em massa verde $\left(163,0 \mathrm{t} \mathrm{MV} \mathrm{ha}^{-1}\right)$ quando comparada aos clones IPA Sertânia (124,3 t MV $\mathrm{ha}^{-1}$ ) e Miúda (117,5 t MV ha $\left.{ }^{-1}\right)$, embora, o mesmo não fosse observado na produtividade em base seca (média de 12,60 t MS ha ${ }^{-1}$ ), e com base na evapotranspiração real acumulada (média de $1.418 \mathrm{~mm}$ ciclo $^{-1}$ ), na qual os três clones não se diferenciaram entre si $(p>0,05)$.

Tendência similar foi verificada para os indicadores de eficiência do uso da água em termos de produtividade da água da cultura $\left(\mathrm{PA}_{\mathrm{C}}\right)$ e de produtividade econômica da água (PEA), usando-se como referência os valores acumulados de precipitação pluviométrica $(\mathrm{P})$ e de evapotranspiração real (ETr), conforme podem ser vistos na tabela 2 .

Em base fresca, a $\mathrm{PA}_{\mathrm{C}(\mathrm{P})}$ entre os clones diferenciaram entre si $(\mathrm{p}<0,05)$, sendo a Orelha de Elefante Mexicana o clone de maior eficiência no uso da água. Todavia, quando se assumiu o volume de água evapotranspirada pelos clones $\left(\mathrm{PA}_{\mathrm{C}(\mathrm{ETT})}\right)$, além da Orelha de Elefante Mexicana, a IPA Sertânia também apresentou altos valores de eficiência. Em ambos os casos, a Miúda foi o clone menos eficiente, por causa da menor produtividade alcançada.

Por outro lado, quando se considerou a $\mathrm{PA}_{\mathrm{C}}$ em base seca não houve diferença significativa entre os clones $(\mathrm{p}>0,05)$, tanto com os valores de P quanto com os de ETr. Assim, a $\mathrm{PA}_{\mathrm{C}}$ com base seca para a palma forrageira durante o período analisado foi de $8,1 \mathrm{~kg} \mathrm{MS} \mathrm{ha}{ }^{-1} \mathrm{~mm}^{-1} \mathrm{em}$ termos de $\mathrm{P}$ e de 9,1 $\mathrm{kg} \mathrm{MS} \mathrm{ha}^{-1} \mathrm{~mm}^{-1}$ em termos de ETr.

Em base fresca, os dados aqui obtidos (86,8 $\mathrm{kg} \mathrm{MV}$ $\mathrm{ha}^{-1} \mathrm{~mm}^{-1}$ para P e $97,1 \mathrm{~kg} \mathrm{MV} \mathrm{ha} \mathrm{mm}^{-1}$ para ETr, independentemente do clone) foram, em média, superiores àqueles citados por Silva et al. (2011) para a cana-deaçúcar (espécie C4) irrigada no Semiárido, que obtiveram 53,6 e 94,9 $\mathrm{kg} \mathrm{MV} \mathrm{ha}^{-1} \mathrm{~mm}^{-1}$, considerando os valores de rendimento industrial de colmos e, nessa ordem, os valores de P + I (P, precipitação; e, I, irrigação) e de ETc (evapotranspiração da cultura).
Com base na ETr, poucos são os dados de $\mathrm{PA}_{\mathrm{C}}$ citados na literatura para a palma. Han e Felker (1997) encontraram, nas condiçóes semiáridas de Kingsville, Texas, USA, para a palma Opuntia ellisiana L., com produção de biomassa seca na ordem de $14,3 \mathrm{t} \mathrm{ha}^{-1}$, eficiência do uso de água igual a $393 \mathrm{~kg} . \mathrm{H}_{2} \mathrm{O} \mathrm{kg} \cdot \mathrm{MS}^{-1}$, equivalente a $25,4 \mathrm{~kg} \mathrm{MS} \mathrm{ha}^{-1} \mathrm{~mm}^{-1}$. Por sua vez, no quarto ano produtivo, quando a produção foi de $17,6 \mathrm{t} \mathrm{ha}^{-1}$, a eficiência atingiu $285 \mathrm{~kg} . \mathrm{H}_{2} \mathrm{O} \mathrm{kg} \cdot \mathrm{MS}^{-1}$, equivalente a $35,1 \mathrm{~kg} \mathrm{MS} \mathrm{ha}^{-1} \mathrm{~mm}^{-1}$.

Nas condições climáticas da Sicília, Itália, região mediterrânea, Consoli et al. (2013) avaliaram a Opuntia ficus-indica L. (Mill.), com plantas de três metros de altura e dez anos de idade, cultivada para fins de produção de fruto, entre junho e setembro de 2009 e em 2010, verificando que a ETr foi de $286 \mathrm{~mm}$ e $252 \mathrm{~mm}$, assim como a eficiência do uso da água de 46,0 kg MS ha ${ }^{-1} \mathrm{~mm}^{-1}$ e 57,0 kg MS ha ${ }^{-1}$ $\mathrm{mm}^{-1}$, respectivamente quanto aos anos avaliados.

$\mathrm{Na}$ maior parte da literatura é comum citar-se o valor de eficiência do uso da palma forrageira com base nos valores de precipitação pluviométrica, sendo ela denominada eficiência do uso da chuva, equivalente à $\mathrm{PA}_{\mathrm{C}}$ com base na precipitação, no presente estudo $\left(\mathrm{PA}_{\mathrm{C}(\mathrm{P})}\right)$, podendo esse valor variar com base em vários aspectos, dentre eles espaçamento, adubação e condiçôes climáticas, e conforme o clone (Dubeux Junior et al., 2006).

Em termos de produtividade econômica da água (PEA), assumindo preços individuais de cladódios e ganho brutos por área para cada clone (tabela 2), percebe-se que não houve diferença estatística significativa entre os clones, tanto em termos de P quanto de ETr. Assim, a PEA da palma forrageira para o período de análise foi, em média, igual a $35,4 \pm 5,4 \mathrm{R} \$ \mathrm{ha}^{-1} \mathrm{~mm}^{-1}$, ou seja, a cultura retorna ao produtor $\mathrm{R} \$ 35,4$ a cada $1 \mathrm{~m}^{3}$ de água que "entra" via precipitação em um hectare do sistema de produção. Por sua vez, em termos de evapotranspiração, ou seja, com base na água que foi efetivamente utilizada pela cultura, esse valor foi de $39,5 \pm 7,7 \mathrm{R} \$ \mathrm{ha}^{-1} \mathrm{~mm}^{-1}$.

Pereira et al. (2012) mostram relaçóes crescentes da PEA com a lâmina de água aplicada no sistema de produçáo de

Tabela 2. Produtividade da água da cultura $\left(\mathrm{PA}_{\mathrm{C}}\right)$ em base seca e fresca, produtividade econômica da água (PEA) com base na água precipitada (Prec.) e na evapotranspiraçáo real da cultura (ETr) e déficit econômico da água $(\triangle \mathrm{EA})$ por três clones de palma forrageira (IPA - IPA Sertânia, MIU - Miúda, OEM - Orelha de Elefante Mexicana) resistentes à Cochonilha do Carmim cultivados, aos dois anos após o plantio, em condições de sequeiro no Semiárido brasileiro

\begin{tabular}{|c|c|c|c|c|c|c|c|}
\hline Ref. & Clone & 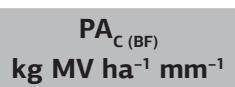 & $\begin{array}{c}\mathrm{PA}_{\mathrm{C(BS)}} \\
\mathrm{kg} \mathrm{MS} \mathrm{ha}^{-1} \mathrm{~mm}^{-1}\end{array}$ & $\begin{array}{c}\text { Preço }^{1 *} \\
\text { R\$ unid. }{ }^{-1}\end{array}$ & $\begin{array}{c}\text { Ganho bruto } \\
\text { R\$ ha-1 }\end{array}$ & $\begin{array}{c}\text { PEA } \\
\mathbf{R} \$ \mathrm{ha}^{-1} \mathrm{~mm}^{-1}\end{array}$ & $\begin{array}{c}\Delta E A \\
R \$ \mathrm{ha}^{-1} \mathrm{~mm}^{-1}\end{array}$ \\
\hline \multirow{3}{*}{ Prec. } & IPA & $80,0 \mathrm{~b}$ & $6,9 \mathrm{a}$ & 0,15 & 57.400 & $36,9 \mathrm{a}$ & 7,4 \\
\hline & MIU & $75,6 \mathrm{~b}$ & $7,4 \mathrm{a}$ & 0,04 & 42.356 & $27,3 \mathrm{a}$ & 0,7 \\
\hline & OEM & $104,8 \mathrm{a}$ & $10,0 \mathrm{a}$ & 0,15 & 65.484 & $42,1 \mathrm{a}$ & 4,1 \\
\hline \multirow{3}{*}{$\mathrm{ETr}$} & IPA & $101,1 \mathrm{AB}$ & $8,8 \mathrm{~A}$ & 0,15 & 57.400 & $44,3 \mathrm{~A}$ & - \\
\hline & MIU & $78,0 \mathrm{~B}$ & 7,7 A & 0,04 & 42.356 & $28,0 \mathrm{~A}$ & - \\
\hline & OEM & $112,1 \mathrm{~A}$ & $10,8 \mathrm{~A}$ & 0,15 & 65.484 & $46,2 \mathrm{~A}$ & - \\
\hline
\end{tabular}

Médias seguidas pela mesma letra minúscula na vertical, usando como referência de cálculo a Prec., e médias seguidas pela mesma letra maiúscula na vertical, usando como referência de cálculo a ETr, não se diferenciam estatisticamente entre si pelo teste de Tukey ao nível de $5 \%$ de significância; Ref. = referência; ${ }^{*}$ Preço da unidade do cladódio destinado à semente segundo o Instituto Agronômico de Pernambuco; as diferenças de preços dos cladódios entre os clones se devem ao seu tamanho, sendo o da Miúda inferior ao tamanho dos cladódios da IPA Sertânia e ao da Orelha de Elefante Mexicana. 
plantas C4 e decrescentes em sistemas com espécies C3. Não se dispóe de dados para espécies MAC. Com base nos valores de $\mathrm{PEA}_{(\mathrm{P})}$ e $\mathrm{PEA}_{(\mathrm{ETr})}\left(35,4 \pm 5,4 \mathrm{R} \$ \mathrm{ha}^{-1} \mathrm{~mm}^{-1}\right.$ e 39,5 \pm 7,7 $\mathrm{R} \$ \mathrm{ha}^{-1} \mathrm{~mm}^{-1}$ ), é possível quantificar outro indicador, denominado neste estudo de déficit econômico da água $\left(\triangle \mathrm{PEA}=\mathrm{PEA}_{(\mathrm{ETT})}-\mathrm{PEA}_{(\mathrm{P})}\right)$, o qual, para condiçóes de sequeiro, reflete quanto o produtor deixou de ganhar em decorrência da ineficiência do sistema de produçáo na utilização do volume de água precipitado. Esse valor foi menor para a Miúda, seguida pela Orelha de Elefante Mexicana, correspondendo a aproximadamente $3 \%$ e $9 \%$ da $\mathrm{PEA}_{(\mathrm{ETr})}$, respectivamente. A IPA Sertânia se mostrou o clone em que o produtor pode deixar de obter maior retorno econômico em decorrência da ineficiência do sistema de produçáo.

Para as condiçóes do Semiárido brasileiro, Teixeira et al. (2007) obtiveram valores de $\mathrm{PEA}_{(\mathrm{P}+\mathrm{I})}$ e $\mathrm{PEA}_{(\mathrm{ETT})}$, em média, iguais a 4,9 US $\$ \mathrm{ha}^{-1} \mathrm{~mm}^{-1}$ e 9,3 US $\$ \mathrm{ha}^{-1} \mathrm{~mm}^{-1}$ para a uva de vinho (equivalente a 9,8 $\mathrm{R} \$ \mathrm{ha}^{-1} \mathrm{~mm}^{-1}$ e $18,6 \mathrm{R} \$$ $\mathrm{ha}^{-1} \mathrm{~mm}^{-1}$, assumindo-se a cotação 1,00 US $\$=\mathrm{R} \$ 2,00$ );

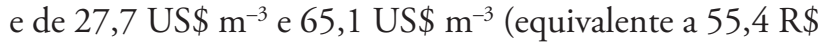
$\mathrm{ha}^{-1} \mathrm{~mm}^{-1}$ e $130,2 \mathrm{R} \$ \mathrm{ha}^{-1} \mathrm{~mm}^{-1}$ ) para a uva de mesa. A partir dos valores citados por esses autores obtêm-se que os valores de $\triangle$ PEA para uva de vinho serão de $8,8 \mathrm{R} \$ \mathrm{ha}^{-1}$ $\mathrm{mm}^{-1}$, aproximadamente $47 \%$, e de $74,8 \mathrm{R} \$ \mathrm{ha}^{-1} \mathrm{~mm}^{-1}$, respondendo por $58 \%$, dos respectivos valores de $\mathrm{PEA}_{(\mathrm{ETr})}$, indicando ineficiência do sistema de produção.

A eficiência do uso da água em termos de nutrientes pode ser vista nas tabelas 3 e 4 . Não se tem referência na literatura quanto a esse indicador de eficiência. Com base nos seus valores é possível analisar quanto de nutrientes foi extraído do solo em relaçáo ao volume de água precipitado ou efetivamente utilizado pela cultura no processo de evapotranspiração.

Em todos os casos, a eficiência em termos de ETr é superior à de precipitaçáo. A diferença entre elas indica a habilidade das plantas em extrair nutrientes do solo com base na água disponível ou mesmo pode representar a perda de água no sistema de produção em relação à absorção de nutrientes. A diferença entre as eficiências com base em $\mathrm{P}$ e ETr, em média, variou de 5\%, para o elemento zinco, a $13 \%$, para o elemento enxofre.

Para os macronutrientes, as eficiências náo se diferenciaram entre si $(\mathrm{p}>0,05)$ (tabela 3), com exceçáo do elemento magnésio com base na evapotranspiração, que foi superior para os clones Orelha de Elefante Mexicana e IPA Sertânia, aqueles que obtiveram maiores magnitudes na produtividade de massa fresca.

O elemento magnésio é um importante constituinte da estrutura da molécula da clorofila e tem papel específico na ativação de enzimas da fotossíntese, da respiração e da síntese de ácidos nucléicos (Taiz e Zeiger, 2004). O aumento da sua absorçáo pela planta está relacionado à concentração dos elementos Ca e K na soluçáo do solo, podendo ele promover o aumento da produtividade das culturas (Frizzone et al., 2005), embora a sua influência varie entre as espécies (Costa et al., 2010).

Tabela 3. Eficiência do uso de macronutrientes (EUN, $\mathrm{g} \mathrm{ha}^{-1} \mathrm{~mm}^{-1}$ ) com base na água precipitada (Prec.) e evapotranspiração (ETr) de três clones de palma forrageira (IPA Sertânia, Miúda e Orelha de Elefante Mexicana) cultivados, resistentes à Cochonilha do Carmim, aos dois anos após o plantio, em condições de sequeiro no Semiárido brasileiro

\begin{tabular}{|c|c|c|c|c|c|c|c|}
\hline Referência & Clone & \multicolumn{6}{|c|}{$\mathrm{g} \mathrm{ha}^{-1} \mathrm{~mm}^{-1}$} \\
\hline \multirow[t]{3}{*}{ Prec. } & IPA & $78,1 \mathrm{a}$ & $3,1 \mathrm{a}$ & 134,7 a & $189,5 \mathrm{a}$ & $60,5 \mathrm{a}$ & $2,2 \mathrm{a}$ \\
\hline & MIU & 85,2 a & $4,0 \mathrm{a}$ & 153,5 a & 243,7 a & $54,3 \mathrm{a}$ & $2,6 \mathrm{a}$ \\
\hline & OEM & $100,0 \mathrm{a}$ & 5,6 a & 229,3 a & 294,5 a & 103,7 a & $2,8 \mathrm{a}$ \\
\hline \multirow[t]{3}{*}{ ETr } & IPA & $99,6 \mathrm{~A}$ & $4,1 \mathrm{~A}$ & $171,9 \mathrm{~A}$ & $244,2 \mathrm{~A}$ & $77,4 \mathrm{AB}$ & $2,8 \mathrm{~A}$ \\
\hline & MIU & $88,0 \mathrm{~A}$ & $4,2 \mathrm{~A}$ & $159,7 \mathrm{~A}$ & $253,8 \mathrm{~A}$ & 55,9 B & $2,8 \mathrm{~A}$ \\
\hline & OEM & $108,4 \mathrm{~A}$ & $6,2 \mathrm{~A}$ & $245,8 \mathrm{~A}$ & $313,3 \mathrm{~A}$ & $111,4 \mathrm{~A}$ & $3,1 \mathrm{~A}$ \\
\hline
\end{tabular}

Médias seguidas pela mesma letra minúscula na vertical, usando como referência de cálculo a Prec., e médias seguidas pela mesma letra maiúscula na vertical, usando como referência de cálculo a ETr, não se diferenciam estatisticamente entre si pelo teste de Tukey ao nível de 5\% de significância.

Tabela 4. Eficiência do uso de micronutrientes (EUN, $\mathrm{mg} \mathrm{ha}^{-1} \mathrm{~mm}^{-1}$ ) com base na água precipitada (Prec.) e evapotranspiração (ETr) de três clones de palma forrageira (IPA Sertânia, Miúda e Orelha de Elefante Mexicana) cultivados, resistentes à Cochonilha do Carmim, aos dois anos após o plantio, em condiçóes de sequeiro no semiárido pernambucano

\begin{tabular}{|c|c|c|c|c|c|c|c|}
\hline Referência & Clone & \multicolumn{5}{|c|}{$\mathrm{mg} \mathrm{ha}^{-1} \mathrm{~mm}^{-1}$} & $\mathrm{EUN}_{(\mathrm{Na})}$ \\
\hline \multirow{3}{*}{ Prec. } & IPA & $210,3 a$ & $24,1 \mathrm{a}$ & $114,58 a$ & $1090,6 \mathrm{a}$ & $76,6 \mathrm{a}$ & $3210,8 b$ \\
\hline & MIU & $225,8 a$ & $67,2 \mathrm{a}$ & 204,47 a & $4554,1 \mathrm{a}$ & $174,6 \mathrm{a}$ & $3383,6 \mathrm{ab}$ \\
\hline & OEM & $315,4 a$ & $23,2 \mathrm{a}$ & $149,31 \mathrm{a}$ & 944,6 a & 392,9 a & $4854,3 \mathrm{a}$ \\
\hline \multirow{3}{*}{$\mathrm{ETr}$} & IPA & $270,6 \mathrm{~A}$ & $32,4 \mathrm{~A}$ & $148,13 \mathrm{~A}$ & $1373,2 \mathrm{~A}$ & $97,5 \mathrm{~A}$ & $4110,6 \mathrm{~A}$ \\
\hline & MIU & $234,6 \mathrm{~A}$ & $70,3 \mathrm{~A}$ & $215,06 \mathrm{~A}$ & $4891,1 \mathrm{~A}$ & $183,4 \mathrm{~A}$ & $3504,8 \mathrm{~A}$ \\
\hline & OEM & $341,1 \mathrm{~A}$ & $24,2 \mathrm{~A}$ & $159,84 \mathrm{~A}$ & $1043,3 \mathrm{~A}$ & $399,1 \mathrm{~A}$ & $5211,5 \mathrm{~A}$ \\
\hline
\end{tabular}

Médias seguidas pela mesma letra minúscula na vertical, usando como referência de cálculo a Prec., e médias seguidas pela mesma letra maiúscula na vertical, usando como referência de cálculo a ETr, nâo se diferenciam estatisticamente entre si pelo teste de Tukey ao nível de 5\% de significância. 
Os valores médios de EUN da palma forrageira para os macronutrientes $\mathrm{N}, \mathrm{P}, \mathrm{K}, \mathrm{Ca}, \mathrm{Mg}$ e $\mathrm{S}$, com base na precipitação, foram respectivamente iguais a $87,8 \mathrm{~g} \mathrm{~N} \mathrm{ha}^{-1}$ $\mathrm{mm}^{-1}$, 4,2 $\mathrm{g} \mathrm{P} \mathrm{ha}^{-1} \mathrm{~mm}^{-1}, 172,5 \mathrm{~g} \mathrm{~K} \mathrm{ha}^{-1} \mathrm{~mm}^{-1}$, 242,6 $\mathrm{g}$ Ca ha ${ }^{-1} \mathrm{~mm}^{-1}$, 72,8 $\mathrm{g} \mathrm{Mg} \mathrm{ha}^{-1} \mathrm{~mm}^{-1}$ e 2,5 g S ha ${ }^{-1} \mathrm{~mm}^{-1}$. Em termos de ETr, por sua vez, os valores médios foram de $98,7 \mathrm{~g} \mathrm{~N} \mathrm{ha}^{-1} \mathrm{~mm}^{-1}$, 4,8 $\mathrm{g} \mathrm{P} \mathrm{ha}^{-1} \mathrm{~mm}^{-1}$, 192,5 $\mathrm{g} \mathrm{K} \mathrm{ha}^{-1}$ $\mathrm{mm}^{-1}$, 270,4 $\mathrm{g} \mathrm{Ca} \mathrm{ha}^{-1} \mathrm{~mm}^{-1}$, 81,6 g Mg ha $\mathrm{gm}^{-1}$ e 2,9 $\mathrm{g} \mathrm{Sha}^{-1} \mathrm{~mm}^{-1}$.

Em termos de eficiência do uso dos micronutrientes com base na precipitação (tabela 4), com exceção da eficiência do uso do elemento $\mathrm{Na}$, para as demais eficiências náo foram verificadas diferenças significativas $(p>0,05)$.

A Orelha de Elefante Mexicana e a Miúda, ou seja, os clones com maiores magnitudes da evapotranspiração, apresentaram maior eficiência no uso do elemento sódio $\left(\mathrm{EUN}_{\left(\mathrm{Na}_{\mathrm{a}}\right)}\right)(\mathrm{p}<0,05)$, entretanto a IPA Sertânia não se diferiu da Miúda. Esse resultado pode ser indicativo de maior resistência, em ambiente semiárido, da espécie do gênero Opuntia sp. em relação às do gênero Nopalea sp., já que o elemento sódio tem grande importância na atividade estomática de espécies MAC (Taiz e Zeiger, 2004), conferindo uma maior eficiência ao uso da água, que no presente estudo foi obtida pela Orelha de Elefante Mexicana, comprovada por seu maior desempenho produtivo em massa fresca.

Para os micronutrientes $\mathrm{B}, \mathrm{Cu}, \mathrm{Fe}, \mathrm{Mn}, \mathrm{Zn}$ e $\mathrm{Na}$, com base na precipitação, as médias de EUN para a palma forrageira foram iguais a $250,5 \mathrm{mg} \mathrm{B} \mathrm{ha}^{-1} \mathrm{~mm}^{-1}, 38,2 \mathrm{mg}$ $\mathrm{Cu} \mathrm{ha}{ }^{-1} \mathrm{~mm}^{-1}$, 1561,2 $\mathrm{mg} \mathrm{Fe} \mathrm{ha}^{-1} \mathrm{~mm}^{-1}$, 2196,4 mg Mn $\mathrm{ha}^{-1} \mathrm{~mm}^{-1}$, 214,7 $\mathrm{mg} \mathrm{Zn} \mathrm{ha}^{-1} \mathrm{~mm}^{-1}$ e 3816,2 mg Na ha ${ }^{-1}$ $\mathrm{mm}^{-1}$. Finalmente, em termos de ETr, suas eficiências foram $282,1 \mathrm{mg} \mathrm{B} \mathrm{ha}^{-1} \mathrm{~mm}^{-1}, 42,3 \mathrm{mg} \mathrm{Cu} \mathrm{ha}{ }^{-1} \mathrm{~mm}^{-1}, 1743,4 \mathrm{mg}$ $\mathrm{Fe} \mathrm{ha}^{-1} \mathrm{~mm}^{-1}$, 2435,9 $\mathrm{mg} \mathrm{Mn} \mathrm{ha}^{-1} \mathrm{~mm}^{-1}$, 226,7 $\mathrm{mg} \mathrm{Zn} \mathrm{ha}^{-1}$ $\mathrm{mm}^{-1}$ e 4275,6 $\mathrm{mg} \mathrm{Na} \mathrm{ha}^{-1} \mathrm{~mm}^{-1}$.

\section{CONCLUSÃO}

A Orelha de Elefante Mexicana e a IPA Sertânia são os clones que se destacaram em termos de eficiência do uso da água considerando a produção de massa fresca. Em termos de massa seca, os clones apresentam a mesma eficiência de uso da água, tanto em termos de água precipitada quanto de evapotranspirada.

Os clones avaliados são similares quanto à produtividade econômica da água e eficiência do uso de nutrientes, com exceção da eficiência do uso do elemento magnésio, com base na evapotranspiração, que foi maior para Orelha de Elefante Mexicana e IPA Sertânia, e do elemento sódio, com base na precipitação, que foi superior nos clones Orelha de Elefante Mexicana e Miúda.

\section{AGRADECIMENTOS}

Ao CNPq e à Facepe, pelo apoio financeiro. Ao Instituto Agronômico de Pernambuco, pela concessão da área experimental. À Embrapa Semiárido, pela disponibilização do conjunto Diviner@2000 para monitoramento do conteúdo de água no solo, pelas análises físicas do solo e pelas de composição mineral e bromatológicas dos clones de palma forrageira. À Universidade Federal Rural de Pernambuco, pela liberação da infraestrutura que possibilitou a realização desta pesquisa.

\section{REFERÊNCIAS}

ALI, M.H.; HOQUE, M.R.; HASSAN, A.A.; KHAIR, A. Effects of deficit irrigation on yield, water productivity and economic returns of wheat. Agricultural water management, v.92, p.151-161, 2007. http://dx.doi.org/10.1016/j.agwat.2007.05.010

ALLEN, R.G.; PEREIRA, L.S.; RAES, D.; SMUTH, M. Crop Evapotranpiration: Guidelines for computing crop water requirements, Rome: FAO, 1998. 301p. (Irrigation and Drainage Paper, n.56).

AMARAL, J.F.T.; MARTINEZ, H.E.P.; LAVIOLA, B.G; FERNANDES FILHO, E.I.; CRUZ, C.D. Eficiência de utilização de nutrientes por cultivares de cafeeiro. Ciência Rural, v.41, p.621-629, 2011. http:// dx.doi.org/10.1590/S0103-84782011005000027

ARAYA, A.; STROOSNIJDER, L.; GIRMAY, G.; KEESSTRA, S.D. Crop coefficient, yield response to water stress and water productivity of teff (Eragrostis tef(Zucc.). Agricultural Water Management, n.98, p.775-783, 2011. http://dx.doi.org/10.1016/j.agwat.2010.12.001

AZEVEDO, P.V.; SOUZA, C.B.; SILVA, B.B.; SILVA, V.P.R. Water requirements of the pineapple crop grown in tropical environments, Brazil. Agricultural Water Management, v.88, p.201-208, 2007. http://dx.doi.org/10.1016/j.agwat.2006.10.021

CONSOLI, S.; INGLESE, G.; INGLESE, P. Determination of evapotranspiration and annual biomass productivity of a cactus pear (Opuntia ficus-indica L. (Mill.) orchard in a Semi-arid Environment. Journal of Irrigation and Drainage Engineering, v.139, p.680-690, 2013. http://dx.doi.org/10.1061/(ASCE)IR.1943-4774.0000589

COSTA, S.A.D.; QUEIROGA, F.M.; PEREIRA, F.H.F.; MARACAJÁ, P.B.; SOUSA FILHO, A.L. Efeito de doses de magnésio na produção e qualidade de frutos de meláo. Revista Verde de Agroecologia e Desenvolvimento Sustentável, v.5, p.118-123, 2010.

DI PAOLO, E.; RINALDI, M. Yield response of corn to irrigation and nitrogen fertilization in a Mediterranean environment. Field Crops Research, v.105, p.202-210, 2008. http://dx.doi.org/10.1016/j. fcr.2007.10.004

DUBEUX JUNIOR, J.C.B.; SANTOS, M.V.F.; LIRA, M.A.; SANTOS, D.C.; FARIAS, I.; LIMA, L.E.; FERREIRA, R.L.C. Productivity of Opuntia ficus-indica (L.) Miller under different N and P fertilization and plant population in north-east Brazil. Journal of Arid Environments, v.67, p.357-372, 2006. http://dx.doi.org/10.1016/j. jaridenv.2006.02.015 
EMPRESA BRASILLEIRA DE PESQUISA AGROPECUÁRIA - EMBRAPA. Sistema Brasileiro de Classificação de Solos. Brasília: EMBRAPA/SPI, 1999. 412p.

FRIZZONE, J.A.; CARDOSO, S.S.; REZENDE, R. Produtividade e qualidade de frutos de meloeiro cultivado em ambiente protegido com aplicação de dióxido de carbono e de potássio via água de irrigação. Acta Scientiarum Agronomy, v.27, p.707-717, 2005.

GEERTS, S.; RAES, D. Deficit irrigation as an on-farm strategy to maximize crop water productivity in dry areas. Agricultural Water Management, v.96, p.1275-1284, 2009. http://dx.doi.org/10.1016/j. agwat.2009.04.009

HAN, H.; FELKER, P. Field validation of water-use efficiency of the CAM plant Opuntia ellisiana in south Texas. Journal of Arid Environments, v.36, p.133-148, 1997. http://dx.doi.org/10.1006/ jare. 1996.0202

IGBADUN, H.E., MAHOO, H.F.; TARIMO, A.K.P.R.; SALIM, BA.S. Crop water productivity of an irrigated maize crop in Mkoji sub-catchment of the Great Ruaha River Basin, Tanzania. Agricultural Water Management, v.85, p.141-150, 2006. http://dx.doi.org/10.1016/j. agwat.2006.04.003

JALOTA, S.K.; SOOD, A.; CHAHAL, G.B.S.; CHOUDHURY, B.U. Crop water productivity of cotton (Gossypium hirsutum L.) wheat (Triticum aestivum L.) system as influenced by deficit irrigation, soil texture and precipitation. Agricultural water management, v.84, p.137-146, 2006. http://dx.doi.org/10.1016/j.agwat.2006.02.003

KARAM, F.; LAHOUD, R.; MASAAD, R.; KABALAN, R.; BREIDI, J.; CHALITA, C.; ROUPHAEL, Y. Evapotranspiration, seed yield and water use efficiency of drip irrigated sunflower under full and deficit irrigation conditions. Agricultural water management, v.90, p.213-223, 2007. http://dx.doi.org/10.1016/j.agwat.2007.03.009

KO, J.; PICCINNI, G. Corn yield responses under crop evapotranspiration-based irrigation management. Agricultural Water Management, v.96, p.799-808, 2009. http://dx.doi.org/10.1016/j. agwat.2008.10.010

LIBARDI, P.L. Dinâmica da água no solo. São Paulo: Editora da Universidade de São Paulo, 2005. p.41-277. (Acadêmica, n.61).

LÓPEZ-URREA, R.; OLALLA, F.M.S.; MONTORO, A.; LÓPEZFUSTER, P. Single and dual crop coefficients and water requirements for onion (Allium cepa L.) under semiarid conditions. Agricultural Water
Management, v.96, p.1031-1036, 2009. http://dx.doi.org/10.1016/j. agwat.2009.02.004

NOBEL, P.S.; BOBICH, E.G. Environmental biology. In: NOBEL, P. (Ed.). Cacti: Biology and Uses. Berkeley: University of California Press, 2002. 280p.

OLIVEIRA, F.T.; SOUTO, J.S.; SILVA, R.P.; ANDRADE FILHO, F.C.; PEREIRA JÚNIOR, E.B. Palma forrageira: Adaptação e importância para os ecossistemas áridos e semiáridos. Revista Verde de Agroecologia e Desenvolvimento Sustentável, v.5, p.27-37, 2010.

PEREIRA, L.S.; CORDERY, I.; IACOVOS, I. Improved indicators of water use performance and productivity for sustainable water conservation and saving. Agricultural Water Management, v.108, p.39-51, 2012. http://dx.doi.org/10.1016/j.agwat.2011.08.022

SALES, A.T.; ANDRADE, A.P.; SILVA, D.S.; VIEIRA, M.L.M.; VIANA, B.L.; LEÓN, M.J.; SÓLIS, A.M. Adaptation potential of cactus pear to soil and climatic conditions of the Semi-Arid in Paraiba State, Brazil. Acta Horticulturae, v.811, p.395-400, 2009.

SENTEK. Calibration of Sentek Pty Ltd soil moisture sensors. Stepney: Sentek Pty Ltd, 2001.

SENTEK. Diviner 2000: user guide version 1.21. Stepney: Sentek Pty Ltd, 2000.

SILVA, T.G.F.; MOURA, M.S.B.; ZOLNIER, S.; SOARES, J.M.; VIEIRA, V.J.S.; FARIAS JÚNIOR, W.G. Demanda hídrica e eficiência do uso de água da cana-de-açúcar irrigada no semiárido brasileiro. Revista Brasileira de Engenharia Agrícola e Ambiental, v.15, p.12571265, 2011. http://dx.doi.org/10.1590/S1415-43662011001200007

SILVA, D.J.; QUEIROZ, A.C. Análises de alimentos (métodos químicos e biológicos). 3.ed. Viçosa: Editora UFV, 2002. 235p.

SUYKER, A.E.; VERMA, S.B. Evapotranspiration of irrigated and rainfed maize-soybean cropping systems. Agricultural and Forest Meteorology, v.149, p.443-452, 2009. http://dx.doi.org/10.1016/j. agrformet.2008.09.010

TAIZ, L.; ZEIGER, E. Fisiologia Vegetal. 3.ed. Porto Alegre: Editora Artmed, 2004. 719p.

TEIXEIRA, A.H.C.; BASTIAANSSEN, W.G.M.; BASSOI, L.H. Crop water parameters of irrigated wine and table grapes to support water productivity analysis in Sao Francisco River basin, Brazil. Agricultural Water Management, v.94, p.31-42, 2007. http://dx.doi. org/10.1016/j.agwat.2007.08.001 